\title{
Inefficient jet-induced star formation in Centaurus A
}

\section{High resolution ALMA observations of the northern filaments ${ }^{\star} \star \star$}

\author{
Q. Salomé ${ }^{1}$, P. Salomé2 ${ }^{2}$ M.-A. Miville-Deschênes ${ }^{1}$, F. Combes ${ }^{2,3}$, and S. Hamer ${ }^{4}$ \\ ${ }^{1}$ Institut d'Astrophysique Spatiale, CNRS, Univ. Paris-Sud, Université Paris-Saclay, Bâtiment 121, 91405 Orsay Cedex, France \\ e-mail: quentin.salome@ias.u-psud.fr \\ 2 LERMA, Observatoire de Paris, PSL Research Univ., CNRS, Sorbonne Univ., UPMC Univ. Paris 06, 75014 Paris, France \\ 3 Collège de France, 11 place Marcelin Berthelot, 75005 Paris, France \\ ${ }^{4}$ CRAL, Observatoire de Lyon, CNRS, Université Lyon 1, 9 avenue Ch. André, 69561 Saint-Genis Laval Cedex, France
}

Received 23 June 2017 / Accepted 27 October 2017

\begin{abstract}
NGC 5128 (Centaurus A) is one of the best targets to study AGN feedback in the local Universe. At $13.5 \mathrm{kpc}$ from the galaxy, optical filaments with recent star formation lie along the radio jet direction. This region is a testbed for positive feedback, here through jet-induced star formation. Atacama Pathfinder EXperiment (APEX) observations have revealed strong CO emission in star-forming regions and in regions with no detected tracers of star formation activity. In cases where star formation is observed, this activity appears to be inefficient compared to the Kennicutt-Schmidt relation. We used the Atacama Large Millimeter/submillimeter Array (ALMA) to map the ${ }^{12} \mathrm{CO}(1-0)$ emission all along the filaments of NGC 5128 at a resolution of $1.3^{\prime \prime} \sim 23.8$ pc. We find that the CO emission is clumpy and is distributed in two main structures: (i) the Horseshoe complex, located outside the HI cloud, where gas is mostly excited by shocks and where no star formation is observed, and (ii) the Vertical filament, located at the edge of the HI shell, which is a region of moderate star formation. We identified 140 molecular clouds using a clustering method applied to the CO data cube. A statistical study reveals that these clouds have very similar physical properties, such as size, velocity dispersion, and mass, as in the inner Milky Way. However, the range of radius available with the present ALMA observations does not enable us to investigate whether or not the clouds follow the Larson relation. The large virial parameter $\alpha_{\text {vir }}$ of the clouds suggests that gravity is not dominant and clouds are not gravitationally unstable. Finally, the total energy injection in the northern filaments of Centaurus A is of the same order as in the inner part of the Milky Way. The strong CO emission detected in the northern filaments is an indication that the energy injected by the jet acts positively in the formation of dense molecular gas. The relatively high virial parameter of the molecular clouds suggests that the injected kinetic energy is too strong for star formation to be efficient. This is particularly the case in the horseshoe complex, where the virial parameter is the largest and where strong $\mathrm{CO}$ is detected with no associated star formation. This is the first evidence of AGN positive feedback in the sense of forming molecular gas through shocks, associated with low star formation efficiency due to turbulence injection by the interaction with the radio jet.
\end{abstract}

Key words. methods: data analysis - galaxies: individual: Centaurus A - galaxies: evolution - galaxies: interactions galaxies: star formation - radio lines: galaxies

\section{Introduction}

Active galactic nuclei (AGN) are assumed to play a major role in regulating star formation. The so-called AGN negative feedback is often invoked to explain the small number of massive galaxies compared to the predictions of the $\Lambda$ cold dark matter (CDM) model (Bower et al. 2006; Croton et al. 2006; Harrison et al. 2012; Dubois et al. 2013; Werner et al. 2014). On the contrary, AGN with pronounced radio jets are prone to positive feedback (Zinn et al. 2013). In particular, in high-redshift radio galaxies, optical emission was found to be aligned with the radio morphology. Such alignment is interpreted as the result of overpressured clouds in the intergalactic medium from the radio jet, triggering star formation in the direction of the radio jet (McCarthy et al. 1987; Begelman \& Cioffi 1989; Rees 1989; de Young 1989). Evidence

\footnotetext{
* This paper makes use of the following ALMA data ADS/JAO.ALMA\#2015.1.01019.S.

$\star \star$ The full Table A.1 and a catalogue of the molecular clouds are only available at the CDS via anonymous ftp to

cdsarc.u-strasbg.fr (130.79.128.5) or via

http://cdsarc.u-strasbg.fr/viz-bin/qcat?J/A+A/608/A98
}

of so-called jet-induced star formation was recently found at low (van Breugel et al. 2004; Feain et al. 2007; Inskip et al. 2008; Elbaz et al. 2009; Reines et al. 2011; Combes 2015) and high redshift (Klamer et al. 2004; Miley \& de Breuck 2008; Emonts et al. 2014). Recent studies were also conducted with numerical simulations to study the effect of radio jets on star formation in the host galaxy or along the jet direction (Wagner et al. 2012; Gaibler et al. 2012; Bieri et al. 2016; Fragile et al. 2017).

The widely studied nearby galaxy NGC 5128 (also known as Centaurus A) is the perfect target to study the radio jetgas interaction. This galaxy hosts a massive disc of dust, gas, and young stars in its central regions (Israel 1998) and is surrounded by faint arc-like stellar shells (at a radius of several kpc around the galaxy) in which HI gas has been detected (Schiminovich et al. 1994). Aligned with the radio jet, CO emission has been observed in the gaseous shells (Charmandaris et al. 2000). In addition, Auld et al. (2012) detected a large amount of dust $\left(\sim 10^{5} M_{\odot}\right)$ around the northern shell region.

Optically bright filaments are observed in the direction of the radio jet (Blanco et al. 1975; Graham \& Price 1981; Morganti et al. 1991). Galaxy Evolution Explorer (GALEX) data (Auld et al. 2012) and young stellar clusters 
(Mould et al. 2000; Rejkuba et al. 2001) indicate that star formation occurs in these filaments. These so-called inner and outer filaments are located at a distance of $\sim 7.7 \mathrm{kpc}$ and $\sim 13.5 \mathrm{kpc}$ from the central galaxy, respectively. The inner and outer filaments show distinct kinematical components, i.e. a well-defined knotty filament and a more diffuse structure, as highlighted by optical excitation lines (VIMOS and MUSE; Santoro et al. 2015a,b; Hamer et al. 2015). Recently Santoro et al. (2016) identified a star-forming cloud in the MUSE data that contains several HII regions. Some of these regions are currently forming stars, whereas star formation seems to have recently stopped in the others.

In Salomé et al. (2016a), we mapped the outer filaments (hereafter the northern filaments) in ${ }^{12} \mathrm{CO}(2-1)$ with APEX. The molecular gas was found to be very extended with a surprisingly $\mathrm{CO}$ bright region outside the HI cloud. We found that star formation does not occur in this $\mathrm{CO}$ bright region. In the other part of the filaments, star formation appears to be inefficient compared to the Kennicutt-Schmidt law (Salomé et al. 2016b). However, the jet-gas interaction seems to trigger the atomic-to-molecular gas phase transition (Salomé et al. 2016a), suggesting that positive feedback is occuring in the filaments.

The goal of this paper is to understand why the molecular gas in the northern filaments of Centaurus A does not follow the Kennicutt-Schmidt law, and whether there is a difference between the eastern $\mathrm{CO}$ bright region and the rest of the filaments. To do so, we decided to observe the $\mathrm{CO}$ emission seen with APEX at high resolution in order to resolved GMCs. In this paper, we present recent ALMA observations of the ${ }^{12} \mathrm{CO}(1-0)$ line along the northern filaments of Centaurus A. The data and a method to extract clouds are presented in Sect. 2. In Sect. 3, we analyse the data and conduct a statistical analysis of the clouds. We discuss our results in Sect. 4 and conclude in Sect. 5.

\section{From $\mathrm{CO}$ emission to individual clouds}

\subsection{ALMA data}

We mapped the ${ }^{12} \mathrm{CO}(1-0)$ emission in the northern filaments of Centaurus A with the $12 \mathrm{~m}$ array of the Atacama Large Millimeter/submillimeter Array (ALMA) during Cycle 3 using Band 3 receivers (project ADS/JAO.ALMA\#2015.1.01019.S). The map covers a region of $6.1^{\prime} \times 4.3^{\prime}$ and consists in a mosaic of 34 pointings, each with an integration time between 140 and $430 \mathrm{~s}$. The baselines ranged from $15 \mathrm{~m}$ to $704 \mathrm{~m}$, providing a resolution of $1.30^{\prime \prime} \times 0.99^{\prime \prime}\left(\mathrm{PA}=81.5^{\circ}\right)$. At the distance of Centaurus A (3.8 Mpc, 1" 18.3 pc; Harris et al. 2010), this corresponds to a beam size of $23.8 \times 18.1 \mathrm{pc}$. The largest angular scale recovered by the interferometer is $14^{\prime \prime}$, which corresponds to about $260 \mathrm{pc}$ at the distance of Centaurus A.

The data were calibrated using the Common Astronomy Software Applications (CASA) and the supplied script. Owing to the primary beam correction, the noise level of the map is not uniform and higher on the edge of map. The histogram of the noise level peaks at $6.5 \mathrm{mJy} /$ beam at a spectral resolution of $1.47 \mathrm{~km} \mathrm{~s}^{-1}$.

\subsection{Cloud identification method}

To identify clouds in the ALMA data, we first used a Gaussian decomposition method developed by Miville-Deschênes et al. (2017). This algorithm allows recovery of the signal, even at low signal-to-noise ratio. Clustering is then made with a threshold descent similar to the clumpfind algorithm (Williams et al. 1994), but applied on a cube of the integrated flux (see Miville-Deschênes et al. 2017, for the details).
We kept the structures with a size larger or equal to the beam size. We also discarded those with central velocities outside the range $-350 \leq v_{\text {cent }} \leq-100 \mathrm{~km} \mathrm{~s}^{-1}$ (where $\mathrm{CO}(2-1)$ has been detected; Salomé et al. 2016a) or velocity dispersion higher than $\sigma_{v} \sim 50 \mathrm{~km} \mathrm{~s}^{-1}$. The clustering method we used enables us to reconstruct a data cube of the modelled signal. We then applied the clumpfind algorithm (Williams et al. 1994) on this cube with five equally spaced threshold values from 6.5 to $58.5 \mathrm{mJy} / \mathrm{beam}$. After deleting spurious clumps smaller than the beam, we obtained a catalogue of 140 GMCs. The map of the integrated CO intensity (Fig. 1) shows small bright spots that are likely associated with barely resolved giant molecular clouds (GMCs). Figure 1 also shows the maps of the central velocity and velocity dispersion of the GMCs.

\subsection{Effect of large-scale filtering}

In Salomé et al. (2016a), we observed the ${ }^{12} \mathrm{CO}(2-1)$ line with APEX along the northern filaments of Centaurus A. We determined that the total molecular gas mass is $(9.8 \pm 0.6) \times 10^{7} M_{\odot}$. With ALMA, the mass derived from the ${ }^{12} \mathrm{CO}(1-0)$ emission is much smaller $2.5 \times 10^{7} M_{\odot}$; i.e. $1.73 \times 10^{7} M_{\odot}$ when we exclude three structures that lie outside the region previously observed with APEX. In particular, in the $\mathrm{CO}$ bright region discovered by Salomé et al. (2016a), the total mass recovered by ALMA is $1.24 \times 10^{7} M_{\odot}$, a factor of 5 smaller than the mass derived from the $\operatorname{CO}(2-1)$ from $\operatorname{APEX}(6.3 \pm 0.2) \times 10^{7} M_{\odot}$.

Such a difference can be partly explained by short-spacing filtering. The northern filaments have also been mapped with the Atacama Compact Array (ACA) during Cycle 3. These data will be presented in a future paper, however we already determined that the molecular gas mass is a factor of 4 higher than that derived with the data from the ALMA $12 \mathrm{~m}$ array alone. The difference of mass between ALMA and APEX may also result from the $\mathrm{CO}(2-1) / \mathrm{CO}(1-0)$ ratio used to derive the mass with APEX (0.55; following Charmandaris et al. 2000). Taking into account the factor of 4 due to the short-spacing filtering, we estimate that the $\mathrm{CO}(2-1) / \mathrm{CO}(1-0)$ ratio is about $0.7-0.8$. This will be the subject of a third forthcoming paper, where we will compare the $\mathrm{CO}(1-0)$ emission from ALMA with high- $J$ CO transitions observed with APEX in the CO bright region, and discuss the excitation of gas in this region.

\section{Results}

\subsection{Spatial distribution of molecular clouds}

Figure 1 shows the maps of the $\mathrm{CO}(1-0)$ intensity, central velocity, and velocity dispersion of the clouds extracted with the method presented in Sect. 2.2. The distribution shows that most of the gas is distributed in low filling factor structures over the whole region, at the present noise level $\left(\mathrm{rms} \sim 113 \mathrm{mJy} \mathrm{km} \mathrm{s}^{-1}\right.$ ), as suggested by the three distinct unresolved and dynamically separated clumps previously found in archival ALMA CO(2-1) data (Salomé et al. 2016b). The intensity map reveals the clumpy structure of molecular gas in the northern filaments of Centaurus A from which we could identify different complexes.

As expected from previous APEX data (Salomé et al. 2016a), most of the $\mathrm{CO}(1-0)$ emission comes from the eastern part of the filaments (almost $77 \%$ of the mass). In this region, the velocity map (Fig. 1 - bottom left) shows the possible existence of coherent filamentary structures that present a horseshoe-like shape, along which molecular clouds are distributed. The clouds in the Horseshoe complex have higher 

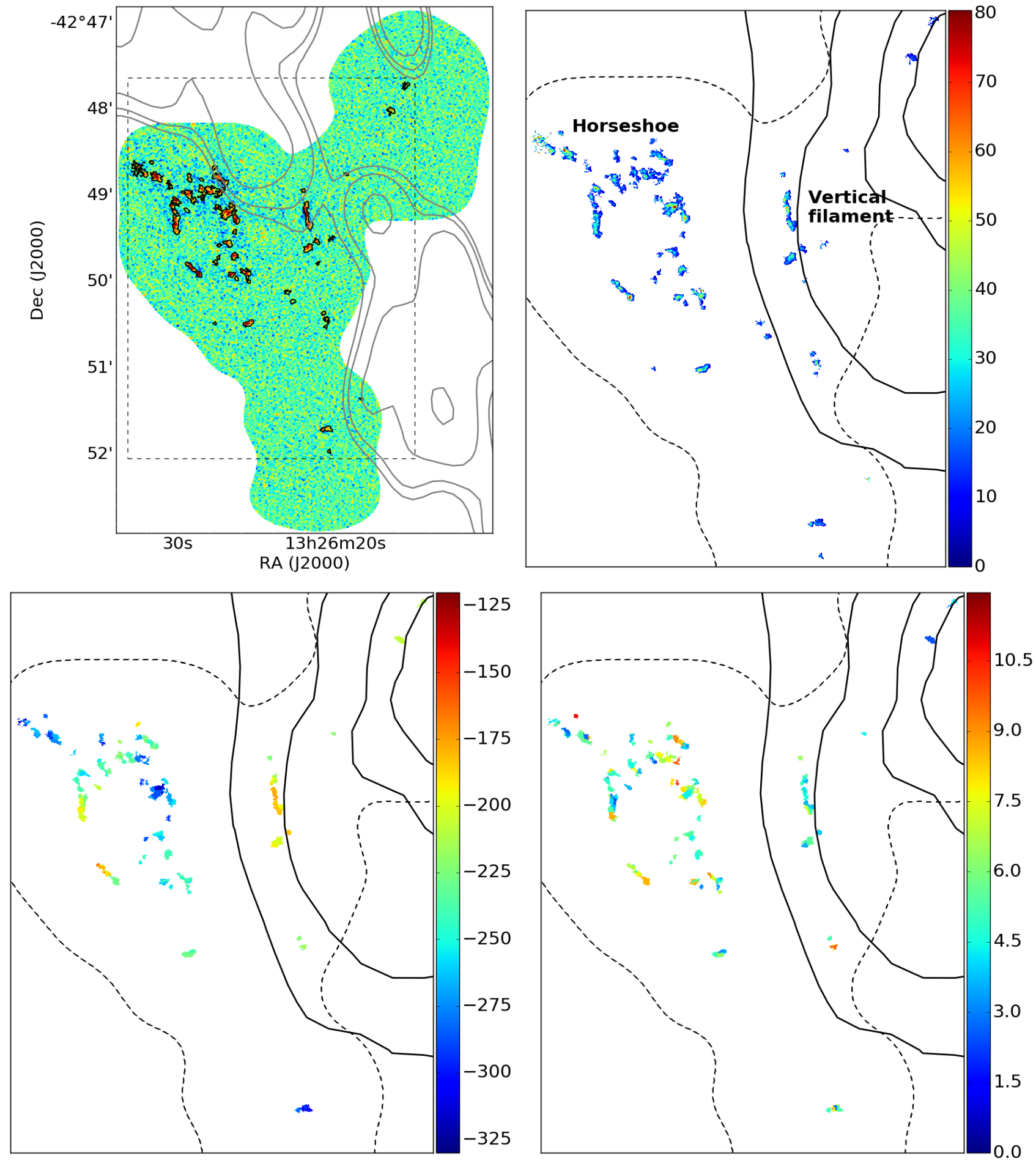

Fig. 1. Top left: map of the moment 0 of the ALMA data produced with CASA. We reported, in black contours, the structures extracted by the method presented in Sect. 2.2. We indicate the location of the radio jet in grey contours. Top right: integrated CO intensity map in mJy km $\mathrm{s}^{-1}$ of the molecular clouds seen in $\mathrm{CO}(1-0)$ with ALMA. Bottom: maps of the central velocity relative to Centaurus A (left) and velocity dispersion (right) in km s${ }^{-1}$ of the clouds. The area of the three maps corresponds to the dashed box in the moment 0 map. The full line and dashed contours represent the HI emission (Schiminovich et al. 1994) and the region observed with ALMA, respectively. The CO emission is clumpy and covers only a small fraction of the region mapped with ALMA.

velocity dispersions (Fig. 1 - bottom right) than in the other molecular clouds of the northern filaments.

To put the $\mathrm{CO}$ emission in context, Fig. 2 presents the spatial relationship with other tracers, namely far-UV (FUV), dust emission, $\mathrm{H} \alpha, \mathrm{HI}$, and young star clusters. In the eastern region, the structure observed in $\mathrm{CO}$ follows the dust emission observed with Herschel with a similar morphology. The
Horseshoe complex is also associated with a similar structure seen in $\mathrm{H} \alpha$ emission. In contrast, there is no FUV emission associated with the Horseshoe complex or young stellar clusters.

While $\mathrm{CO}$ emission covers all the dust emission in the eastern region, it only covers a small fraction of dust emission in the HI cloud. Most of the $\mathrm{CO}$ emission in the HI cloud is distributed in a vertical filament located at the edge of the HI cloud. 

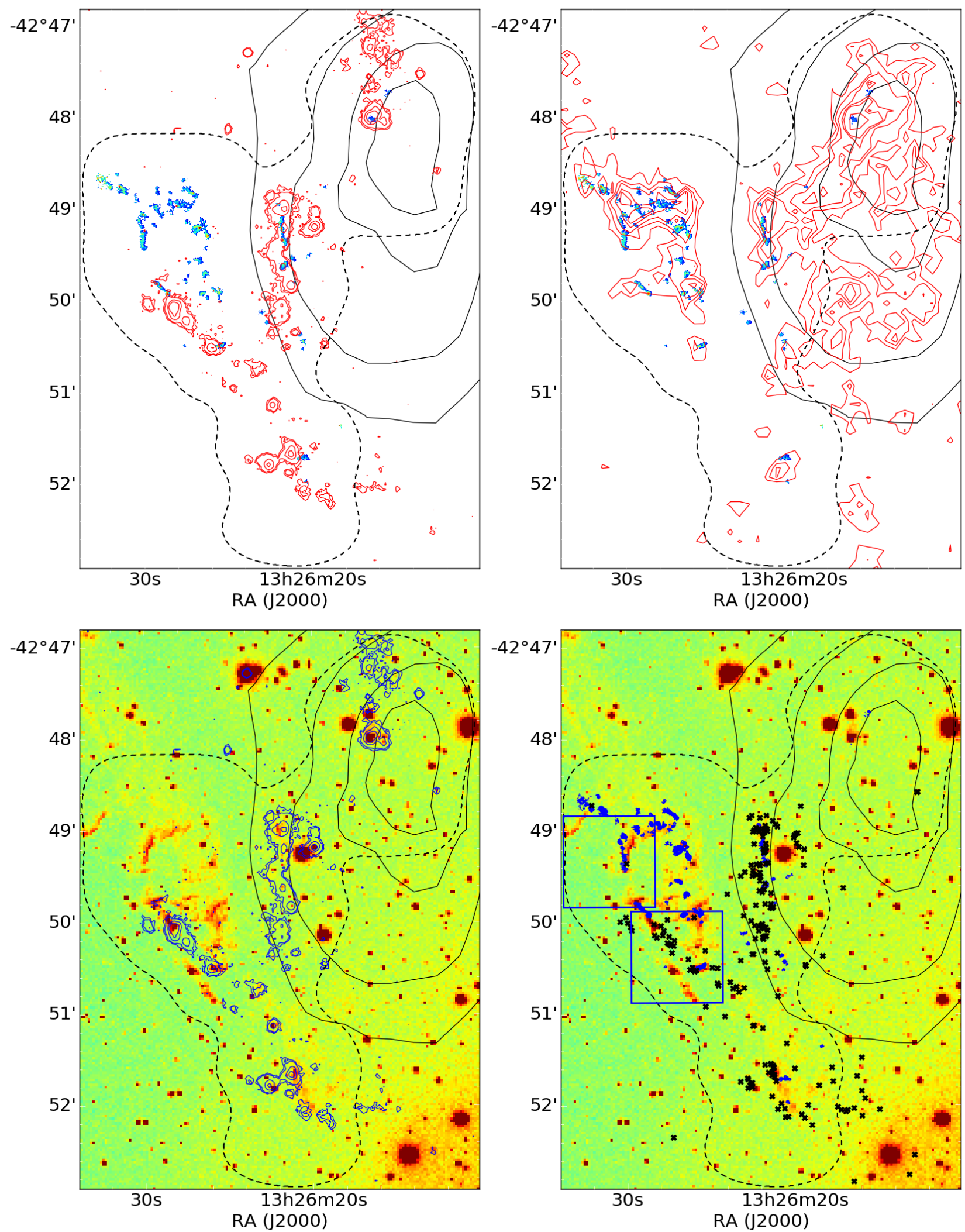

Fig. 2. Distribution of the ALMA CO(1-0) emission in relation to star formation regions. Top: CO intensity map with contours of the GALEX FUV (left - Neff et al. 2015a) and Herschel FIR (right) emission. Bottom: the colour map represents the H $\alpha$ emission seen with CTIO. In all four images, the black contours represent the HI emission and the black dashed line corresponds to the area observed with ALMA. The crosses on the bottom right image indicate the position of the young star clusters from Rejkuba et al. (2001). The Horseshoe complex, which corresponds to the bright CO emission from Salomé et al. (2016a), is not associated with young stellar clusters. In contrast, the Vertical filament (at the edge of the HI shell) is associated with $\mathrm{H} \alpha$, FUV emission, and young stellar clusters (Rejkuba et al. 2001), and is likely forming stars. It is not established whether the northern FUV emission is associated with young stars as it was not included in Rejkuba et al. (2001). 
Table 1. Statistics (mean and mean absolute deviation) of the giant molecular clouds properties.

\begin{tabular}{lcccccc}
\hline \hline Quantity & Horseshoe & SF clouds & M33 & LMC & M51 & MW \\
\hline Radius $(\mathrm{pc})$ & $22.0 \pm 4.1$ & $21.2 \pm 3.3$ & $51 \pm 13$ & $16 \pm 5$ & $48 \pm 14$ & 31.5 \\
$\sigma_{v}\left(\mathrm{~km} \mathrm{~s}^{-1}\right.$ & $5.6 \pm 1.6$ & $4.9 \pm 1.2$ & $3.8 \pm 0.7$ & $1.6 \pm 0.4$ & $6.4 \pm 1.8$ & 4.0 \\
Mass $\left(10^{5} M_{\odot}\right)$ & $1.1 \pm 0.8$ & $1.2 \pm 0.8$ & - & - & - & 1.5 \\
$\Sigma_{H_{2}}\left(M_{\odot} \mathrm{pc}^{-2}\right)$ & $45.1 \pm 18.2$ & $51.3 \pm 21.0$ & $46 \pm 20$ & $21 \pm 9$ & $180 \pm 82$ & 28.6 \\
Density $\left(\mathrm{cm}^{-3}\right)$ & $37.5 \pm 18.4$ & $45.0 \pm 22.1$ & - & - & - & 24.1 \\
$\alpha_{\text {vir }}$ & $12.0 \pm 7.0$ & $8.0 \pm 4.4$ & $2.1 \pm 0.9$ & $1.6 \pm 0.3$ & $1.7 \pm 0.4$ & - \\
\hline
\end{tabular}

Notes. For comparison, we list the properties of clouds in M 33, M 51, the Large Magellanic Cloud, and the Milky Way (Hughes et al. 2013; Miville-Deschênes et al. 2017).

This filament is likely a single coherent structure as it does not show significant difference in the central velocity (Fig. 1). This filament also does not seem to be highly turbulent with velocity dispersions lower than $6-7 \mathrm{~km} \mathrm{~s}^{-1}$. The Vertical filament follows a filament seen in $\mathrm{H} \alpha$ with CTIO (Fig. 2), and is also aligned with FUV emission that is likely produced by young stellar clusters found by Rejkuba et al. (2001). This tends to indicate that the Vertical filament is a region of star formation, contrary to the Horseshoe complex where the $\mathrm{H} \alpha$ emission is mostly excited by shocks (Salomé et al. 2016a).

In addition to the Vertical filament, the HI cloud also contains a few isolated structures seen in CO. In particular, the peak of $\mathrm{HI}$ emission is associated with only two small $\mathrm{CO}$ structures. Interestingly, all the isolated $\mathrm{CO}$ structures within the HI cloud may be star-forming regions as they are associated with FUV emission and young stellar clusters. This also seems to be the case for the $\mathrm{CO}$ emission located in the south that is associated with a bright spot of both FUV and dust emission.

The molecular clouds observed with ALMA seem to present two star formation regimes. Molecular gas in the Horseshoe complex forms stars very inefficiently and the $\mathrm{H} \alpha$ emission is excited by shocks, whereas in the Vertical filament and isolated clouds the $\mathrm{CO}$ emission is associated with recent star formation.

\subsection{Giant molecular clouds}

In this section we discuss the physical properties of the clouds, looking for differences between the Horseshoe complex and starforming regions. In Fig. 3 and Table 1, we report the statistics of the radius, velocity dispersion, mass, surface density, and density.

Size - The angular radius is defined as the emissionweighted radius. We used the implementation made by Miville-Deschênes et al. (2017) that is based on the inertia matrix

$\psi=\left[\begin{array}{cc}\sigma_{\alpha}^{2} & \sigma_{\alpha \delta}^{2} \\ \sigma_{\alpha \delta}^{2} & \sigma_{\delta}^{2}\end{array}\right]$,

where

$$
\begin{aligned}
\sigma_{\alpha}^{2} & =\frac{\sum_{\mathrm{pix}} S_{\mathrm{CO}}^{i}\left(\alpha-\alpha_{i}\right)^{2}}{\sum_{\mathrm{pix}} S_{\mathrm{CO}}^{i}} \\
\sigma_{\delta}^{2} & =\frac{\sum_{\mathrm{pix}} S_{\mathrm{CO}}^{i}\left(\delta-\delta_{i}\right)^{2}}{\sum_{\mathrm{pix}} S_{\mathrm{CO}}^{i}} \\
\sigma_{\alpha \delta}^{2} & =\frac{\sum_{\mathrm{pix}} S_{\mathrm{CO}}^{i}\left(\alpha-\alpha_{i}\right)\left(\delta-\delta_{i}\right)}{\sum_{\mathrm{pix}} S_{\mathrm{CO}}^{i}}
\end{aligned}
$$

with $\alpha, \delta$ the central coordinates of the cloud. The size is given by the eigenvalues of $\psi$, where the largest and smallest half-axis of the cloud $R_{\min }$ and $R_{\max }$ are the maximum and minimum eigenvalues. We assume that the GMCs are prolate and adopt the following definition of the angular radius:

$R_{\text {ang }}=\left(R_{\max } R_{\min } R_{\min }\right)^{1 / 3}$.

Finally, the physical radius is given by $R=D_{\mathrm{L}} \tan \left(R_{\text {ang }}\right)$, where $D_{\mathrm{L}}$ is the luminosity distance. The minimum radius found is limited by the resolution $(R \sim 14.1 \mathrm{pc}$, about 1.4 times the resolution). The distribution of radius is rather narrow; $54 \%$ of the GMCs are larger than twice the resolution and smaller than three times the resolution (Fig. 3 - top left). The larger value of $R$ is $38.4 \mathrm{pc}$, about four times the resolution. This narrow range is due to the way clumpfind identifies structures. Because of lack of larger scale structures in the data (due to interferometry filtering), clumpfind tends to split potentially larger coherent structures in a collection of individual clumps, missing associations. Adding short-spacings will enable us to determine more robustly the spatial-scale distribution of molecular gas.

Velocity dispersion - We defined the central velocity of the clouds as the emission-weighted mean velocity $\langle v\rangle$ and the velocity dispersion $\sigma_{v}$ as follows:

$$
\begin{aligned}
\langle v\rangle & =\frac{\sum_{v} v S_{\mathrm{CO}}(v) \mathrm{d} v}{\sum_{v} S_{\mathrm{CO}}(v)} \\
\sigma_{v} & =\sqrt{\frac{\sum_{v} v^{2} S_{\mathrm{CO}}(v) \mathrm{d} v}{\sum_{v} S_{\mathrm{CO}}(v)}-\langle v\rangle^{2}}
\end{aligned}
$$

where $S_{\mathrm{CO}}(v)$ is the average $\mathrm{CO}$ spectrum of a single cloud, constructed by adding all the voxels of the data cube identified by clumpfind, and $\mathrm{d} v$ is the channel width. The GMCs have velocity dispersions lower than $11 \mathrm{~km} \mathrm{~s}^{-1}$, with an average value of $5.4 \pm 1.4 \mathrm{~km} \mathrm{~s}^{-1}$.

Mass - The CO luminosity was derived using the relation of Solomon et al. (1987),

$L_{\mathrm{CO}}^{\prime}=3.25 \times 10^{7} S_{\mathrm{CO}} \Delta v D_{\mathrm{L}}^{2} v_{\mathrm{obs}}^{-2}(1+z)^{-3}$,

where $\Delta v$ is the FWHM and $S_{\mathrm{CO}} \Delta v=\sum_{i} S_{\mathrm{CO}} \Delta v_{i}$ is the total integrated emission of the cloud. The mass is then estimated by applying a CO-to- $\mathrm{H}_{2}$ conversion factor of $\alpha_{\mathrm{CO}}=$ $4.3 M_{\odot}\left(\mathrm{K} \mathrm{km} \mathrm{s}^{-1} \mathrm{pc}^{2}\right)^{-1}$ (Bolatto et al. 2013). The total mass of the GMCs is $\sim 1.6 \times 10^{7} M_{\odot}$. This represents about $63 \%$ of the mass extracted from the $\mathrm{CO}(1-0)$ ALMA data by the Gaussian decomposition and clustering methods. The mass distribution of the clouds is shown in Fig. 3 (middle left). The mass ranges from $7.8 \times 10^{3}$ to $6.1 \times 10^{5} M_{\odot}$ with a mean value of $1.1 \times 10^{5} M_{\odot}$. We fitted the high mass part of the distribution using a power-law $\mathrm{d} N / \mathrm{d} \log (M) \propto M^{-\alpha}$ assuming a $1 / \sqrt{N}$ uncertainty for each data point. For $M \geq 9.3 \times 10^{4} M_{\odot}$ we obtain $\alpha=0.75 \pm 0.18$. This 

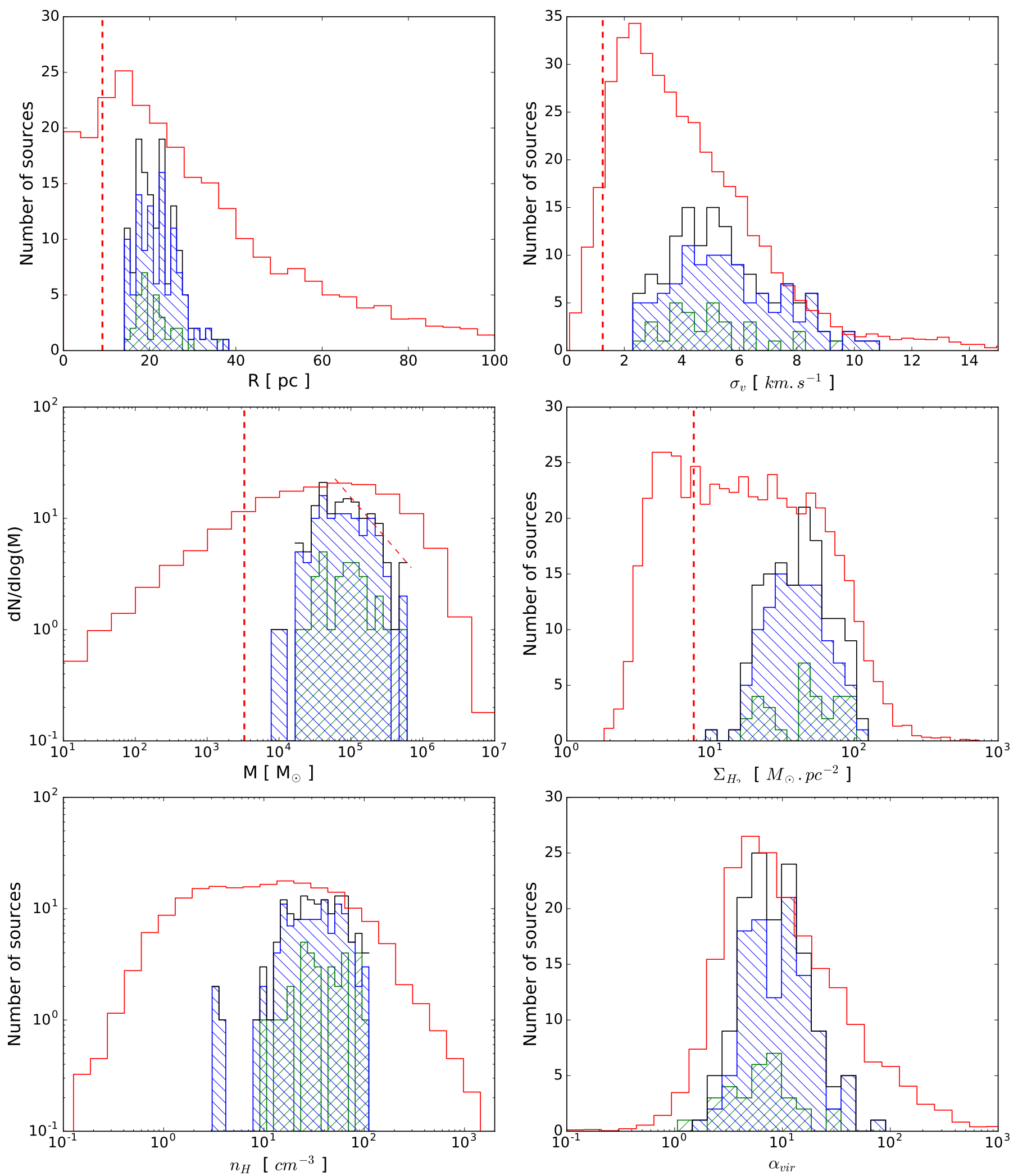

Fig. 3. Histogram of the properties of the giant molecular clouds. Top: characteristic radius and velocity dispersion. Middle: molecular gas mass and surface density. The red dashed line in the left plot is the best fitting power law for masses $M \geq 9 \times 10^{4} M_{\odot}$. Bottom: volume density and virial parameter. The vertical red dashed lines correspond to the lower limits due to resolution or sensitivity. In the six plots, we added the histograms of GMCs of the Milky Way (Miville-Deschênes et al. 2017). The clouds were separated in two groups: (i) the Horseshoe complex in blue, where gas is excited by shocks; and (ii) the star-forming clouds in green, associated with $\mathrm{H} \alpha$, FUV emission, and young stellar clusters.

value is consistent with that found for giant molecular clouds in general $\left(\alpha \sim 0.8\right.$ for $M>10^{4} M_{\odot}$, Solomon et al. 1987; Kramer et al. 1998; Heyer et al. 2001; Marshall et al. 2009).
Surface density - The angular area of a cloud is defined as $A=N_{\text {pix }} \mathrm{d} \Omega$, where $N_{\text {pix }}$ is the number of pixels on the sky and $\mathrm{d} \Omega$ is the solid angle of a single pixel. Dividing the mass 


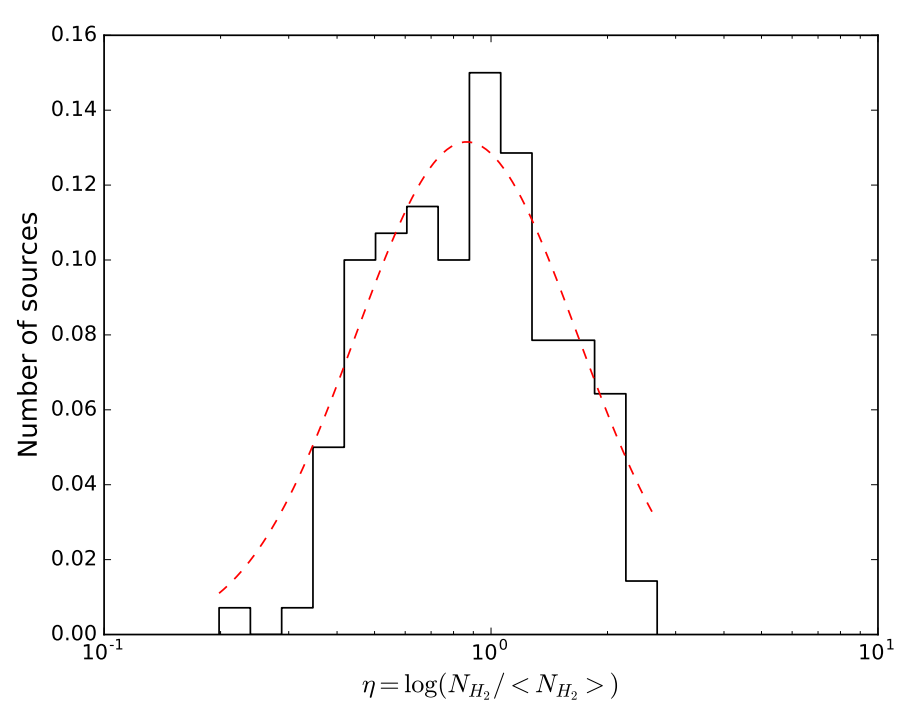

Fig. 4. Probability distribution function of the $\mathrm{H}_{2}$ column density of the clouds. The $x$-axis shows the normalised column density. The red dashed line shows the best fitting log-normal $(\sigma=0.66 \pm 0.08, \mu=$ $0.29 \pm 0.11)$.

by this area in squared parsecs, we obtain the surface density $\Sigma_{\mathrm{H}_{2}}$. The histogram of the GMCs surface density is rather narrow with an average value of $46.5 \pm 19.1 M_{\odot} \mathrm{pc}^{-2}$. In particular, the histogram does not show low surface densities comparable to the outer Milky Way. This is likely due to the sensitivity of the ALMA observations. At the present noise level, the lower limit detectable at $3 \sigma$ is $I_{\mathrm{CO}}>1.75 \mathrm{~K} \mathrm{~km} \mathrm{~s}^{-1}$, which corresponds to a surface density $\Sigma_{\mathrm{H}_{2}}>7.64 M_{\odot} \mathrm{pc}^{-2}$ (the vertical red dashed line on the middle right panel of Fig. 3).

The probability distribution function of the surface density shows a log-normal shape (Fig. 4). The best fitting model has a location parameter $\mu=0.29 \pm 0.11$ and a scale parameter $\sigma=$ $0.66 \pm 0.08$ (see Fig. 4). Using numerical simulations of compressive supersonic flows, Kritsuk et al. (2007, 2011) showed that the surface density histogram is found to be log-normal in the absence of gravity. When including gravity in the simulations, the histogram at high density deviates from log-normal in the form of a power-law tail. In the northern filaments of Centaurus A, the PDF of the GMCs surface density does not show a power-law behaviour at high surface density. This suggests that the contribution of gravitation is small.

Volume density - The gas density of each cloud is defined by

$n_{\mathrm{H}}=\frac{3 M}{4 \pi R^{3}} \frac{1}{\left(\mu+f_{\mathrm{H}_{2}}\right) m_{\mathrm{H}}}$,

where $\mu=2.4$ to take heavy elements into account and $f_{\mathrm{H}_{2}}$ is the molecular gas fraction. As previously found by Salomé et al. (2016a), the filaments are mostly molecular thus, we assume $f_{\mathrm{H}_{2}}=1$. The GMCs cover a large range of densities from 3.0 to $98.8 \mathrm{~cm}^{-3}$ with an average density of $39.2 \pm 19.3 \mathrm{~cm}^{-3}$ (see Fig. 3 - bottom left).

\subsection{Larson's relations}

Mass-size relation - The top left panel of Fig. 5 shows the relation between the mass and the size of the $\mathrm{CO}$ clouds. We added the 2D histograms of the molecular clouds in the Milky Way (Miville-Deschênes et al. 2017). The giant molecular clouds of the northern filaments of Centaurus $\mathrm{A}$ are consistent with the dispersion for molecular clouds in the inner Milky Way. We tried a bisector linear regression $Y$ versus $X$ and $X$ versus $Y$ to estimate the variation of mass with the radius. The index of the power law is larger than that found for the Milky Way (Miville-Deschênes et al. 2017). However, the dispersion of the points in the $M-R$ plot is of the same order as the range of radius available with the present ALMA observations (less than one order of magnitude), therefore fitting a linear relation is not really significant yet.

Velocity-size relation - The $\sigma_{v}-R$ relation is shown in the top right panel of Fig. 5. For this plot, the data points also agree with the 2D histogram of the Milky Way distribution. However, there is no correlation between $\sigma_{v}$ and $R$ for the molecular clouds of the northern filaments. Again, this is likely due to the small coverage in the spatial scale of the present observations. In addition to $\sigma_{v}-R$, we explored various relations between $\sigma_{v}$ and other cloud parameters. The velocity dispersion and the mass are well correlated (Pearson coefficient of 0.66), as well as $\sigma_{v}$ and $(\Sigma R)$, with a Pearson coefficient of 0.68 .

Spatial frequencies - We found that the GMCs identified with clumpfind in the northern filaments of Centaurus A have physical properties distributed in the range of the values found for the Milky Way. However, ALMA does not enable us to fully explore this. As an interferometer, ALMA only recovered the spatial frequencies between 1.3" and 14" (from 18.1 to $260 \mathrm{pc}$ ). It is now essential to add observations at higher resolution to reach cloud radii smaller than $10 \mathrm{pc}$ and short-spacing observations for radii larger than $260 \mathrm{pc}$. We plan to revisit the Larson relations for the large structures in a forthcoming paper in which we will combine ALMA data with our recent ACA observations.

\subsection{Excitation of the clouds}

In this section, we focus on the fields of view previously observed in the northern filaments with MUSE (Santoro et al. $2015 b$ ). Observations include the principal excitation optical lines $\mathrm{H} \alpha \lambda 6562.8$, [NII] $\lambda 6583, \mathrm{H} \beta \lambda 4861.3$, [OIII] $\lambda 4959,5007$, [OI] $\lambda 6366$, and the two [SII] $\lambda 6716,6731$ lines. Figure 7 shows the velocity map of the $\mathrm{H} \alpha$ and $\mathrm{CO}$ emission, with the same colour scale. By simply comparing the colour of the maps at the location of the $\mathrm{CO}$ structures, we clearly found that $\mathrm{CO}$ is blueshifted with respect to $\mathrm{H} \alpha$. A pixel-by-pixel comparison shows that the velocity $\mathrm{CO}$ is on average blueshifted by $v_{\mathrm{CO}}-v_{\mathrm{H} \alpha}=-35.3 \pm 23.2 \mathrm{~km} \mathrm{~s}^{-1}$.

We computed pixel-by-pixel BPT diagrams based on the $\mathrm{H} \alpha,[\mathrm{NII}], \mathrm{H} \beta,[\mathrm{OIII}],[\mathrm{OI}]$, and [SII] lines from MUSE (Fig. 6; Baldwin et al. 1981; Kewley et al. 2006). Each line was fitted by a Gaussian at each spatial resolution element (see Hamer et al. 2014 , for the details) to measure the flux. Figure 8 shows a map of the different regions regarding the excitation process within the velocity range $-330<v<-120 \mathrm{~km} \mathrm{~s}^{-1}$. The structures covered by the MUSE field of views are mostly excited by energy injection from the radio jet or shocks. The small inclusion excited by star formation claimed by Santoro et al. (2016) is spatially coincident with one $\mathrm{CO}$ structure.

\section{Discussion}

Stability of the clouds - For each GMC extracted from the ALMA data, we derived the free-fall time $t_{\mathrm{ff}}$ and the dynamical time $t_{\mathrm{dyn}}$ of the clouds. These timescales are defined by

$$
\begin{aligned}
t_{\mathrm{ff}} & =4.4 \times 10^{7}\left(\frac{n}{\mathrm{~cm}^{-3}}\right)^{-1 / 2} \mathrm{yr} \\
t_{\mathrm{dyn}} & =9.8 \times 10^{5}\left(\frac{R}{\mathrm{pc}}\right)\left(\frac{\sigma_{v}}{\mathrm{~km} \mathrm{~s}^{-1}}\right)^{-1} \mathrm{yr} .
\end{aligned}
$$



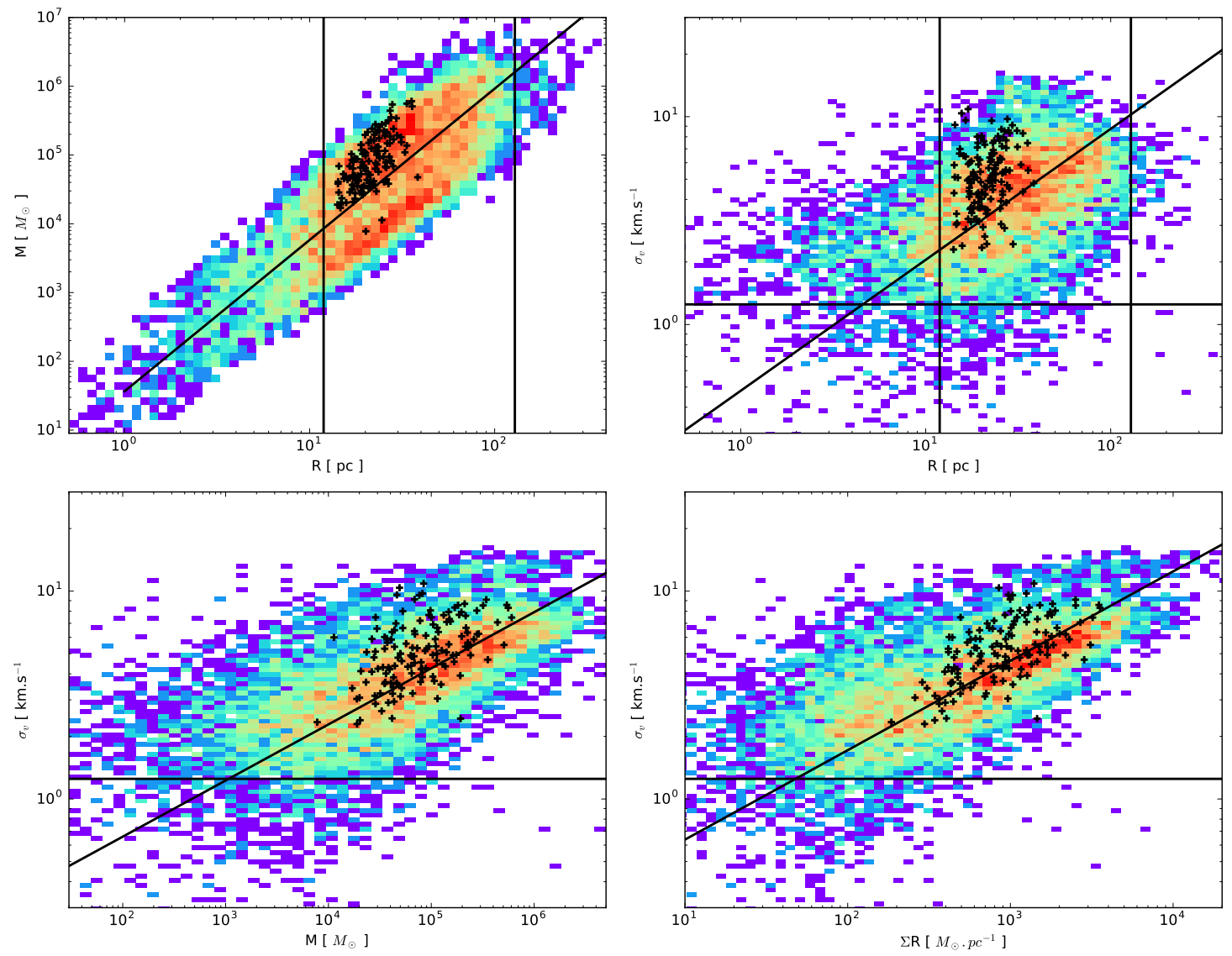

Fig. 5. Plot of the Larson's relations for the giant molecular clouds (black points). The top row shows the $M-R($ left) and $\sigma-R($ right $)$ relations. The bottom row shows the $\sigma-M$ (left) and $\sigma-\Sigma R$ (right) relations. We also plot the 2D histograms and the best fitting relations (colour scale and black line) for the molecular clouds in the Milky Way (Miville-Deschênes et al. 2017). The molecular clouds in the northern filaments of Centaurus A are consistent with those of the Milky Way. We also indicate the limits on the radius and velocity dispersion that can be reached owing to resolution or filtering effects $\left(18.1 \leq R \leq 260 \mathrm{pc}, \sigma_{v}>1.25 \mathrm{~km} \mathrm{~s}^{-1}\right.$; horizontal and vertical black lines).

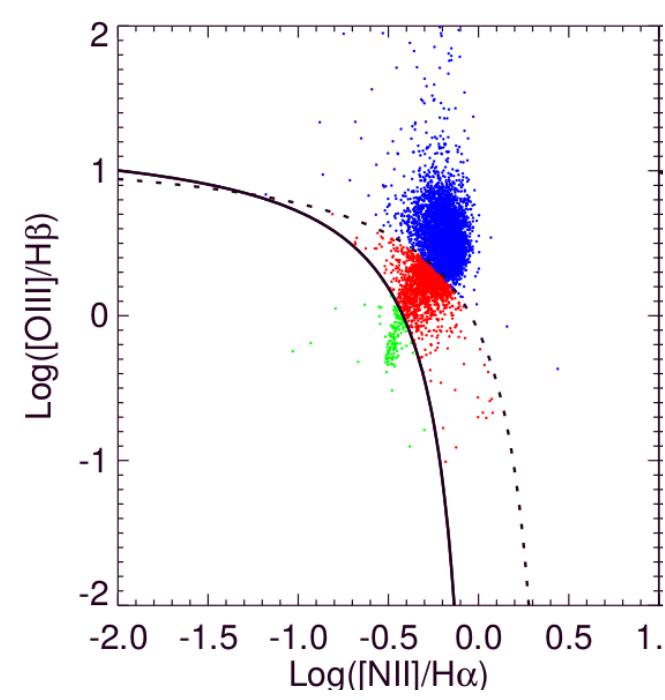

Fig. 6. Pixel-by-pixel BPT diagrams of the CO clumps with MUSE. The black line represents the empirical separation of star formation (green) and AGN/shock-ionised regions (blue). The dotted line shows the extreme upper limit for star formation (Kewley et al. 2006). The red points correspond to the composite regime.

The free-fall time is about twice as long as the dynamical time, i.e. $t_{\text {dyn }} \sim(4.4 \pm 1.2) \times 10^{6} \mathrm{yr}$ and $t_{\mathrm{ff}} \sim(8.3 \pm 2.3) \times 10^{6} \mathrm{yr}$. Equivalently, this translates into a virial parameter, $\alpha_{\mathrm{vir}}=$ $5 \sigma_{v}^{2} R /(G M) \sim t_{\mathrm{ff}}^{2} / t_{\mathrm{dyn}}^{2}$, with an average value of $11.1 \pm 6.6$.
This suggests that the molecular clouds in the northern filaments of Centaurus A are not gravitationally bounded structures. We separated the clouds in two groups: (i) the Horseshoe complex, where gas is excited by shocks, and (ii) the star-forming clouds, associated with $\mathrm{H} \alpha$, FUV emission and young stellar clusters. The histogram of the virial parameter does not show any bimodality (Fig. 3) however, the virial parameter tends to be smaller in the star-forming clouds than in the Horseshoe complex.

Pressure - The internal pressure of the clouds is given by

$$
P_{\mathrm{int}}=\rho_{\mathrm{g}} \sigma_{v}^{2}=4.01 \times 10^{-14}\left(\frac{n}{\mathrm{~cm}^{-3}}\right)\left(\frac{\sigma_{v}}{\mathrm{~km} \mathrm{~s}^{-1}}\right)^{2} \mathrm{dyn} \mathrm{cm}^{-2},
$$

where $\rho_{\mathrm{g}}$ is the molecular gas volume density and $\sigma_{v}$ is the velocity dispersion of the clouds. On average, we found an internal pressure $P_{\text {int }}=(6.0 \pm 4.7) \times 10^{-11} \mathrm{dyn} \mathrm{cm}^{-2}$. Based on X-ray emission, Kraft et al. (2009), Croston et al. (2009) derived ISM pressures of $\sim 10^{-12} \mathrm{dyn} \mathrm{cm}^{-2}$ around the inner radio lobes and the northern middle lobe. In the X-ray knots observed by XMMNewton in the northern middle lobe, a thermal pressure has been estimated to be of the order of $\sim 10^{-11} \mathrm{dyn} \mathrm{cm}^{-2}$ (Kraft et al. 2009). Based on these values, Neff et al. (2015b) derived lower limits of the pressure for the radio features observed at $327 \mathrm{MHz}$ with the VLA. For the diffuse radio emission, these authors estimated that the pressure is of the order of $0.5-1 \times 10^{-11} \mathrm{dyn} \mathrm{cm}^{-2}$. They also found similar values for the radio knots related to 
Q. Salomé et al.: The northern filaments of Centaurus A with ALMA
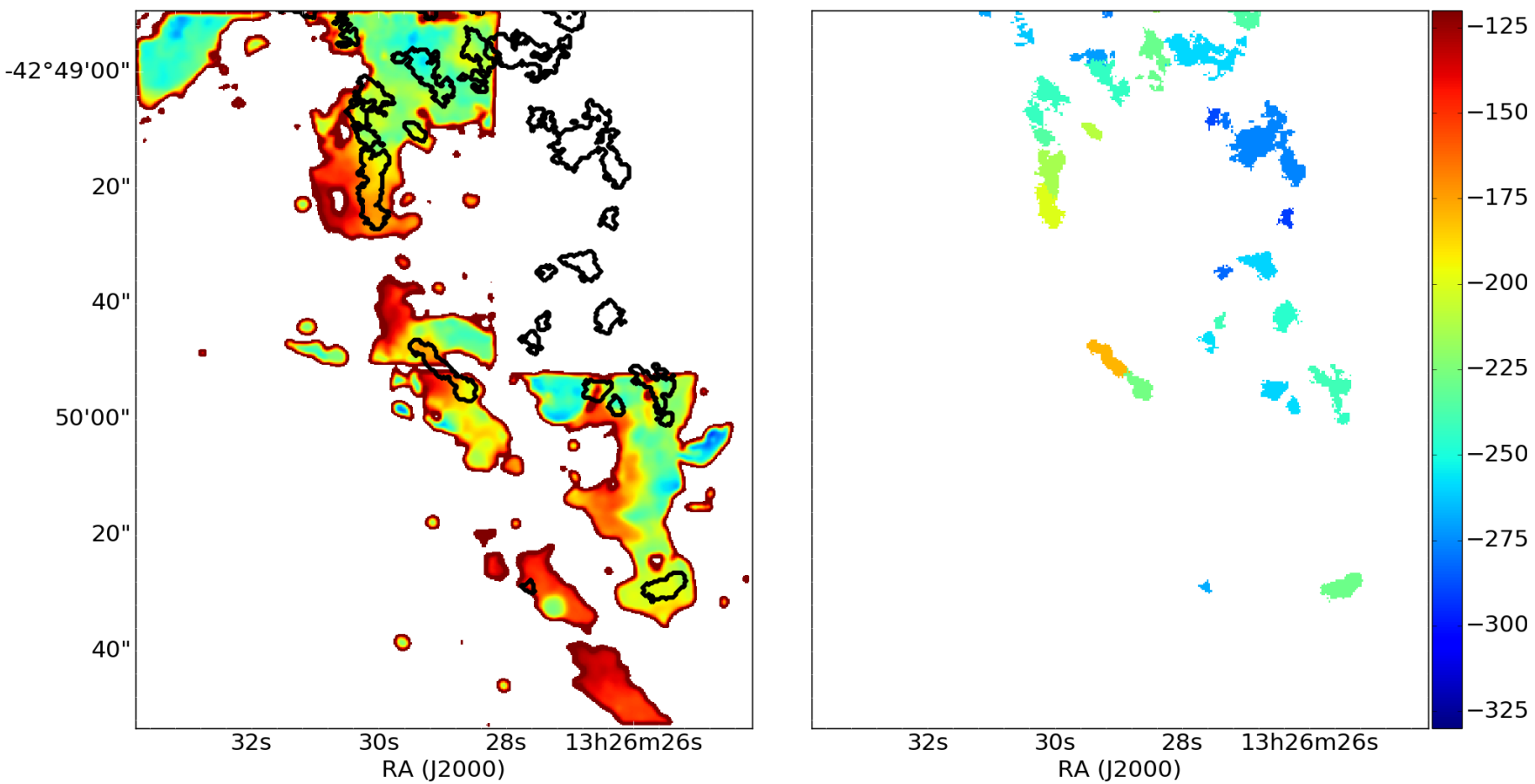

Fig. 7. Velocity maps in $\mathrm{km} \mathrm{s}^{-1}$ of the $\mathrm{H} \alpha$ (left) and $\mathrm{CO}$ emission (right). The black contours in the left panel represent the ALMA CO(1-0) emission distribution. The colour scale is the same for both maps, with a velocity range $-330<v<-120 \mathrm{~km} \mathrm{~s}^{-1}$, relative to Centaurus A. It is thus clear that the $\mathrm{CO}$ is blueshifted compared to the $\mathrm{H} \alpha$ emission.
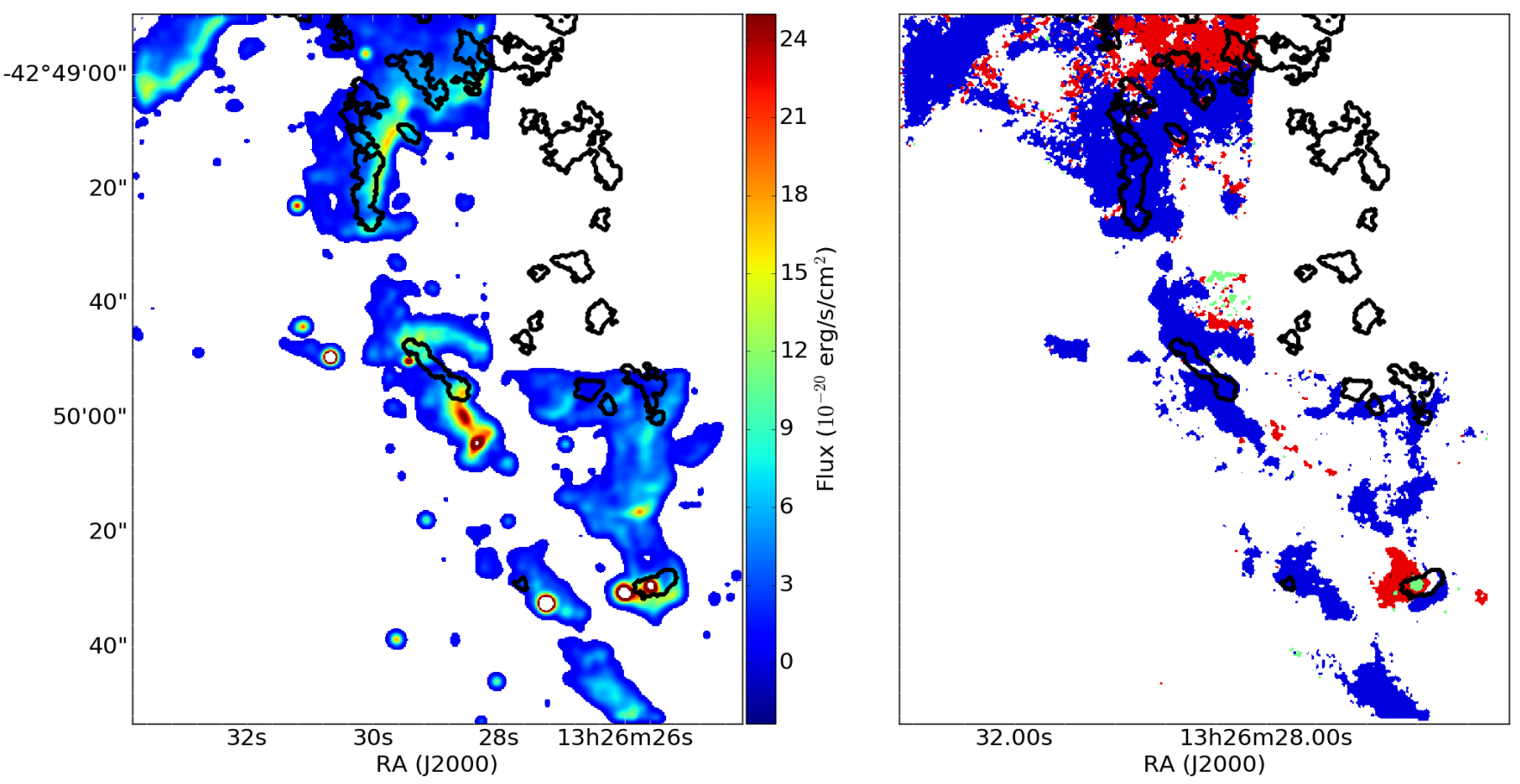

Fig. 8. Maps of the $\mathrm{H} \alpha$-[NII] flux from MUSE (left) and the excitation processes of the CO structures (right). The black contours represent the ALMA CO $(1-0)$ emission distribution. The BPT diagram was computed for the velocity range $-330<v<-120 \mathrm{~km} \mathrm{~s}^{-1}$. Star formation is represented in green and AGN or shocks are indicated in blue. Red corresponds to a composite of star formation and AGN/shocks. The CO clouds in the Horseshoe complex are associated with shock-excited $\mathrm{H} \alpha$ emission, except for one clump that is associated with the star-forming region from Santoro et al. (2016) and young stellar clusters.

the X-ray knots from Kraft et al. (2009). However, the pressures derived by Neff et al. (2015b) are parametrised by the pressure scaling factor $\eta$. The internal pressure we derived for the $\mathrm{CO}$ clouds in the northern filaments of Centaurus A is larger than that estimated from X-ray and radio emission (Kraft et al. 2009; Croston et al. 2009; Neff et al. 2015b). Such a difference might be explain by a pressure scaling factor higher that previously estimated ( $\eta \sim 10$; Neff et al. 2015b). Moreover, the previous estimates of the pressure are average values over a large region. It is likely that the pressure is locally higher. One example is the inner radio lobes where the radio pressure is one order of magnitude higher than in the outer parts (Neff et al. 2015b). 
Energy injection rate - Following Miville-Deschênes et al. (2017), we evaluated the energy injection rate in the clouds. It has been seen numerically (see review by Hennebelle \& Falgarone 2012) that turbulent energy decays in one dynamical time if it is not maintained. Therefore the energy dissipation rate is simply the kinetic energy divided by the dynamical time. Here we make the assumption that turbulence has reached a steady state where energy injection is balanced by energy dissipation. This is corroborated by Neff et al. (2015b) who argued that energy is frequently injected into the outer radio lobes on timescales of the order of $10 \mathrm{Myr}$, comparable to the dynamical time measured here $(\sim 4 \mathrm{Myr})$. In that case the energy injection rate is:

$\dot{E}_{\text {inj }}=-\frac{1}{2} \frac{M \sigma_{v}^{3}}{R}$.

We found $\dot{E}_{\text {inj }}=(1.1 \pm 1.2) \times 10^{2} L_{\odot}$, which is a value similar to what is observed in the inner part of the Milky Way where stars are forming actively (Miville-Deschênes et al. 2017).

General scenario - The comparison of the physical properties of the molecular clouds in the northern filaments with those seen in the Milky Way is instructive. Even though the molecular clouds in the northern filaments have physical properties, such as size, mass, and velocity dispersion, close to what is seen in the Milky Way, there is a fundamental difference when studied in detail. It appears that the virial parameter is key. In the Horseshoe region, where no star formation is detected, the virial parameter has a rather broad distribution peaking at $\alpha_{\text {vir }}=12.0 \pm 7.0$ (see Fig. 3 and Table 1). This distribution is similar to that of molecular clouds seen in the outer part of the Milky Way, where the star formation efficiency is also low. Miville-Deschênes et al. (2017) argued that the formation of molecular clouds in the outer Milky Way could be related to the dynamical action of infalling matter from the Galactic halo and not by self-gravity or stellar feedback in the disc. The molecular clouds in the Horseshoe region could have a similar origin, whereas in this case the dynamical action of the jet would provide the extra pressure to make the gas transits to the cold phase.

Interestingly, the virial parameter of the star-forming regions of the northern filament (Fig. 3 bottom right, green curve) peaks at a lower value around $\alpha_{\text {vir }}=8$, which indicates that self-gravity has a more important dynamical role for these clouds compared to the Horseshoe region. On the other hand, $\alpha_{\text {vir }}$ is still higher on average than in the inner Milky Way, where it peaks at values between 4 and 5 (Miville-Deschênes et al. 2017). This could explain why the star formation efficiency in the northern filaments of Centaurus A is smaller than in the Milky Way.

These results indicate that energy injected in a system by an external source (infall or AGN feedback for instance) can trigger the formation of molecular gas and formation of stars, but at a level lower than that seen in disc galaxies. This suggests that the Schmidt-Kennicutt relation would only apply to a self-regulated system in which self-gravity and stellar feedback are in balance. In other situations, the surface density of molecular gas alone is not a good proxy for the star formation rate. Finally, the presence of FUV emission indicates the presence of a population of $\sim 100 \mathrm{Myr}$ stars and suggests different evolutionary stages for the Horseshoe complex and the Vertical filament. These differences could be due to the history of the local kinetic energy injection by the expanding radio jet. However, it is still possible that stars are forming in the Horseshoe complex if they are obscured by dust.

\section{Conclusion}

In this article, we have presented observations of the ${ }^{12} \mathrm{CO}(1-0)$ in the northern filaments of Centaurus $\mathrm{A}$ at high resolution with the Atacama Large Millimeter/submillimeter Array (ALMA). The region mapped with ALMA corresponds to the region observed with APEX by Salomé et al. (2016a). The resolution of ALMA $\left(1.3^{\prime \prime} \sim 23.8 \mathrm{pc}\right)$ reveals the clumpy structure of the molecular gas, as previously predicted by Salomé et al. (2016b). However, the present data recover only $20 \%$ of the total molecular gas mass found by APEX. Such a difference is likely due to spatial filtering by the interferometer. Recent observations with the ACA reveals that most of the molecular gas is distributed in more extended structures (Salomé et al., in prep.).

We used the Gaussian decomposition and clustering methods developed by Miville-Deschênes et al. (2017) to extract the signal from the data. The $\mathrm{CO}$ emission follows the morphology of the $\mathrm{H} \alpha$ emission. We identified two structures in the northern filaments. First, the Horseshoe complex in the bright $\mathrm{CO}$ region discovered by Salomé et al. (2016a) is associated with $\mathrm{H} \alpha$ emission but not with FUV emission and young stellar clusters. A pixel-by-pixel BPT diagram with optical emission lines from MUSE shows that the CO clouds covered by the MUSE field of views are mostly excited by energy injection from the radio jet or shocks. Second, the Vertical filament is located at the edge of the HI cloud. Aligned with $\mathrm{H} \alpha$, FUV emission, and young stellar clusters, the Vertical filament is likely a region of star formation.

Applying the clumpfind algorithm on the result of the Gaussian decomposition, we extracted 140 molecular clouds. These clouds have size, velocity dispersion, and mass of the same order than molecular clouds in the Milky Way. The range of radius available with the present ALMA observations (less than one order of magnitude) does not enable us to investigate whether the clouds follow the Larson relation or not.

We derived an estimate of the internal pressure of the clouds. On average, we found an internal pressure of $(6.0 \pm 4.7) \times$ $10^{-11} \mathrm{dyn}^{-2}$. This is about one order of magnitude higher that what was derived using X-ray (Kraft et al. 2009; Croston et al. 2009) and radio emission (Neff et al. 2015b). However, the pressures derived by these authors are average values over a large region and pressure is likely higher locally.

Finally, we found that the free-fall time is about twice the dynamical time. This indicates that the molecular clouds are not gravitationally unstable. The derived rate of kinetic energy injected in molecular clouds is similar to the typical value found in the inner Milky Way (Miville-Deschênes et al. 2017). However, the star formation rate in the filaments of Centaurus A is much lower than that in the Milky Way. This suggests that, while the energy injected by the jet-gas interaction triggers the HI-to- $\mathrm{H}_{2}$ phase transition, it is high enough to limit gravitational collapse, especially in the Horseshoe complex where no star formation is observed.

Acknowledgements. We thank the referee for his/her comments. We thank Edwige Chapillon and the IRAM ARC node for their help during the Phase 2, and Marina Rejkuba for providing the catalogue of stellar clusters. ALMA is a partnership of ESO (representing its member states), NSF (USA) and NINS (Japan), together with NRC (Canada), NSC and ASIAA (Taiwan), and KASI (Republic of Korea), in cooperation with the Republic of Chile. The Joint ALMA Observatory is operated by ESO, AUI/NRAO and NAOJ. This work was supported by the ANR grant LYRICS (ANR-16-CE31-0011).

\section{References}

Auld, R., Smith, M. W. L., Bendo, G., et al. 2012, MNRAS, 420, 1882 Baldwin, J. A., Phillips, M. M., \& Terlevich, R. 1981, PASP, 93, 5 
Begelman, M. C., \& Cioffi, D. F. 1989, ApJ, 345, L21

Bieri, R., Dubois, Y., Silk, J., Mamon, G. A., \& Gaibler, V. 2016, MNRAS, 455, 4166

Blanco, V. M., Graham, J. A., Lasker, B. M., \& Osmer, P. S. 1975, ApJ, 198, L63

Bolatto, A. D., Wolfire, M., \& Leroy, A. K. 2013, ARA\&A, 51, 207

Bower, R. G., Benson, A. J., Malbon, R., et al. 2006, MNRAS, 370, 645

Charmandaris, V., Combes, F., \& van der Hulst, J. M. 2000, A\&A, 356, L1

Combes, F. 2015, Galaxies in 3D across the Universe, Proc. Int. Astron., IAU Symp., 309, 182

Croston, J. H., Kraft, R. P., Hardcastle, M. J., et al. 2009, MNRAS, 395, 1999

Croton, D. J., Springel, V., White, S. D. M., et al. 2006, MNRAS, 365, 11

de Young, D. S. 1989, ApJ, 342, L59

Dubois, Y., Gavazzi, R., Peirani, S., \& Silk, J. 2013, MNRAS, 433, 3297

Elbaz, D., Jahnke, K., Pantin, E., Le Borgne, D., \& Letawe, G. 2009, A\&A, 507, 1359

Emonts, B. H. C., Norris, R. P., Feain, I., et al. 2014, MNRAS, 438, 2898

Feain, I. J., Papadopoulos, P. P., Ekers, R. D., \& Middelberg, E. 2007, ApJ, 662, 872

Fragile, P. C., Anninos, P., Croft, S., Lacy, M., \& Witry, J. W. L. 2017, Phys. Rev. D, 91, 043

Gaibler, V., Khochfar, S., Krause, M., \& Silk, J. 2012, MNRAS, 425, 438

Graham, J. A., \& Price, R. M. 1981, ApJ, 247, 813

Hamer, S. L., Edge, A. C., Swinbank, A. M., et al. 2014, MNRAS, 437, 862

Hamer, S., Salomé, P., Combes, F., \& Salomé, Q. 2015, A\&A, 575, L3

Harris, G. L. H., Rejkuba, M., \& Harris, W. E. 2010, PASA, 27, 457

Harrison, C. M., Alexander, D. M., Mullaney, J. R., et al. 2012, ApJ, 760, L15

Hennebelle, P., \& Falgarone, E. 2012, A\&ARv, 20, 55

Heyer, M. H., Carpenter, J. M., \& Snell, R. L. 2001, ApJ, 551, 852

Hughes, A., Meidt, S. E., Colombo, D., et al. 2013, ApJ, 779, 46

Inskip, K. J., Villar-Martín, M., Tadhunter, C. N., et al. 2008, MNRAS, 386, 1797

Israel, F. 1998, A\&ARv, 8, 237

Kewley, L. J., Groves, B., Kauffmann, G., \& Heckman, T. 2006, MNRAS, 372, 961

Klamer, I. J., Ekers, R. D., Sadler, E. M., \& Hunstead, R. W. 2004, ApJ, 612, L97
Kraft, R. P., Forman, W. R., Hardcastle, M. J., et al. 2009, ApJ, 698, 2036

Kramer, C., Stutzki, J., Rohrig, R., \& Corneliussen, U. 1998, A\&A, 329, 249 Kritsuk, A. G., Norman, M. L., Padoan, P., \& Wagner, R. 2007, ApJ, 665, 416 Kritsuk, A. G., Norman, M. L., \& Wagner, R. 2011, ApJ, 727, L20

Marshall, D. J., Joncas, G., \& Jones, A. P. 2009, ApJ, 706, 727

McCarthy, P. J., van Breugel, W., Spinrad, H., \& Djorgovski, S. 1987, ApJ, 321, L29

Miley, G., \& de Breuck, C. 2008, A\&ARv, 15, 67

Miville-Deschênes, M., Murray, N., \& Lee, E. J. 2017, ApJ, 834, 57

Morganti, R., Robinson, A., Fosbury, R. A. E., et al. 1991, MNRAS, 249, 91

Mould, J. R., Ridgewell, A., Gallagher III, J. S., et al. 2000, ApJ, 536, 266

Neff, S. G., Eilek, J. A., \& Owen, F. N. 2015a, ApJ, 802, 88

Neff, S. G., Eilek, J. A., \& Owen, F. N. 2015b, ApJ, 802, 87

Rees, M. J. 1989, MNRAS, 239, 1

Reines, A. E., Sivakoff, G. R., Johnson, K. E., \& Brogan, C. L. 2011, Nature, 470, 66

Rejkuba, M., Minniti, D., Silva, D. R., \& Bedding, T. R. 2001, A\&A, 379, 781

Salomé, Q., Salomé, P., Combes, F., \& Hamer, S. 2016a, A\&A, 595, A65

Salomé, Q., Salomé, P., Combes, F., Hamer, S., \& Heywood, I. 2016b, A\&A, 586, A45

Santoro, F., Oonk, J. B. R., Morganti, R., \& Oosterloo, T. 2015a, A\&A, 574, A89

Santoro, F., Oonk, J. B. R., Morganti, R., Oosterloo, T. A., \& Tremblay, G. 2015b, A\&A, 575, L4

Santoro, F., Oonk, J. B. R., Morganti, R., Oosterloo, T. A., \& Tadhunter, C. 2016, A\&A, 590, A37

Schiminovich, D., van Gorkom, J. H., van der Hulst, J. M., \& Kasow, S. 1994, ApJ, 423, L101

Solomon, P. M., Rivolo, A. R., Barrett, J., \& Yahil, A. 1987, ApJ, 319, 730

van Breugel, W., Fragile, C., Anninos, P., \& Murray, S. 2004, IAUS, 217, 472

Wagner, A. Y., Bicknell, G. V., \& Umemura, M. 2012, ApJ, 757, 136

Werner, N., Oonk, J. B. R., Sun, M., et al. 2014, MNRAS, 439, 2291

Williams, J. P., de Geus, E. J., \& Blitz, L. 1994, ApJ, 428, 693

Zinn, P., Middelberg, E., Norris, R. P., \& Dettmar, R. 2013, ApJ, 774, 66 
A\&A 608, A98 (2017)

\section{Appendix A: Properties of molecular clouds}

Table A.1. Properties of some molecular clouds extracted with the method presented in Sect. 2.2.

\begin{tabular}{lcccccc}
\hline \hline Cloud & $\begin{array}{c}R \\
(\mathrm{pc})\end{array}$ & $\begin{array}{c}S_{\mathrm{CO}} \Delta v \\
\left(\mathrm{Jy} \mathrm{km} \mathrm{s}^{-1}\right)\end{array}$ & $\begin{array}{c}v_{\text {cent }} \\
\left(\mathrm{km} \mathrm{s}^{-1}\right)\end{array}$ & $\begin{array}{c}\Delta v \\
\left(\mathrm{~km} \mathrm{~s}^{-1}\right)\end{array}$ & $\begin{array}{c}M_{\mathrm{H}_{2}} \\
\left(M_{\odot}\right)\end{array}$ & $\begin{array}{c}\Sigma_{\mathrm{H}_{2}} \\
\left(M_{\odot} \mathrm{pc}^{-2}\right)\end{array}$ \\
\hline 1 & 33.39 & 2.909 & -180.97 & 16.65 & $1.90 \times 10^{6}$ & 425.08 \\
2 & 29.31 & 2.338 & -275.31 & 11.01 & $1.52 \times 10^{6}$ & 443.50 \\
3 & 24.55 & 1.591 & -228.34 & 11.58 & $1.04 \times 10^{6}$ & 430.25 \\
4 & 32.94 & 3.579 & -227.82 & 20.20 & $2.33 \times 10^{6}$ & 537.56 \\
5 & 26.90 & 1.841 & -186.42 & 14.60 & $1.20 \times 10^{6}$ & 414.79 \\
6 & 30.44 & 1.907 & -226.01 & 14.67 & $1.24 \times 10^{6}$ & 335.25 \\
7 & 38.33 & 3.359 & -202.54 & 12.97 & $2.19 \times 10^{6}$ & 372.53 \\
8 & 31.30 & 1.271 & -206.29 & 5.73 & $8.28 \times 10^{5}$ & 211.42 \\
9 & 24.70 & 1.101 & -176.68 & 10.77 & $7.18 \times 10^{5}$ & 293.93 \\
10 & 19.60 & 0.774 & -223.42 & 14.97 & $5.04 \times 10^{5}$ & 328.09 \\
\hline
\end{tabular}

Notes. The velocities are relative to Cen A $\left(v_{\mathrm{LSR}} \sim 545 \mathrm{~km} \mathrm{~s}^{-1}\right)$. A catalogue of all the GMCs is available at the CDS. 